\title{
RESUMEN
}

La frambuesa es la especie de mayor importancia económica y comercial entre los berries chilenos, con una superficie plantada cercana a las 10.800 hectáreas. En este contexto, y dado a que la provincia de Ñuble basa gran parte de su economía en la agricultura, la rentabilidad de este mercado y la forma en cómo gestionan suscostos de producción, es de vital importancia para el desarrollo provincial y regional. Por lo anterior, la presente investigación tuvo como objetivo diagnosticar el proceso de administración de costos de los pequeños productores de berries de la provincia de Ñuble, con el fin de determinar puntos críticos y elaborar una propuesta metodológica que les permita un mayor control de sus costos y por ende mejorar su rentabilidad. Desde el punto de vista metodológico se utilizaron técnicas pertenecientes al paradigma de investigación cuantitativo y cualitativo, como encuestas, entrevistas, observación y levantamiento de información en terreno. Los resultados muestran el bajo nivel de registro y control de costos de los productores de frambuesas en la zona, y presenta como propuesta, administrar los costos utilizando el sistema de costeo por procesos, costos históricos y asignando los costos indirectos de fabricación en base a un modelo tradicional basado en el tiempo de duración de cada una de las etapas del proceso productivo.

Palabras Claves: Costos de producción, Producción de frambuesas, Costeo por procesos, Pequeños productores.

\section{SISTEMA DE ADMINISTRACIÓN DE COSTOS EN LA PRODUCCIÓN DE FRAMBUESAS DE PEQUEÑOS PRODUCTORES}

Cecilia Gallegos Muñoz / Estela Rodriguez Quezada²

\section{COST ADMINISTRATION SYSTEM IN RASPBERRIES PRODUCTION OF MINOR PRODUCERS}

\section{ABSTRACT}

The raspberry is the species which has the greatest economical and commercial importance among the chilean berries, with a planted surface of almost 10.800 hectares. In this context, and considering that most of the province of Ñuble's economy is based on agriculture, the profitability of this market and the way in which they manage their production costs is of vital importance for the provincial and regional development. Taking the above into consideration, the current research aimed to diagnose the cost administration system of minor producers of berries in the province of Nuble; so as to determine critical points and develop a methodological proposal which allowed them a greater control of their costs, and in that way improve their profitability. From the methodological point of view, techniques belonging to the quantitative and qualitative paradigm of investigation were used such as surveys, interviews, and on site observation and information collecting. The results show a low level of records and cost control from raspberry producers in the zone. They also present as proposal to administrate the costs using the process costing system, historical costs, and setting aside indirect production costs considering a traditional model based on the time of each stage of the productive process.

Key Words: Production Costs, Raspberry Production, Process Costing System, Minor Producers. 
Revista de Investigación Aplicada en Ciencias Empresariales 


\section{INTRODUCCIÓN}

La provincia de Ñuble basa gran parte de su economía en el área agrícola convirtiéndose en un importante referente de la producción de frambuesas a nivel nacional con un total de 1.493,86 hectáreas sembradas, lo que constituye un $47 \%$ de la superficie total del país, (Odepa, 2017).

Respecto al proceso de producción de frambuesas en la provincia, este considera las siguientes etapas, y es realizado de manera orgánica y convencional:

- Poda: los agricultores podan la planta de la frambuesa

- Control de larva: es realizada luego de la poda de la planta y consiste en aplicar insecticidas a la planta para que ésta no contraiga algún tipo de larva.

- Fertilización: consiste en aplicar a los suelos diversos nutrientes para mejorar el rendimiento del cultivo y la calidad de la fruta.

- Fertilización con nitrógeno: consiste en la aplicación de fertilizantes que contengan nitrógeno para aumentar el vigor de la planta y aumentar la producción de flores y frutos.

- Control de maleza: consiste en la limpieza constante del predio, de las plantas que pueden debilitar el crecimiento de la frambuesa y que crecen de manera rápida y constante alrededor de los cultivos.

- Control de burritos: el burrito es una plaga muy dañina para la planta de frambuesas. Este se anida en el cuello de la planta por lo cual hace que la producción baje. El control de burritos consiste en la aplicación de insecticidas para el manejo y control de esta plaga.

- Fertilización foliar: consiste en la aplicación de fertilizantes pero no directamente al suelo, ya que este se aplica con pulverizadores sobre la planta.

- Control de enfermedades: consiste en el lavado de cañas de la frambuesa, generalmente con cobre para evitar enfermedades de la planta producidas por el agua del riego, bichos, entre otros.

- Control de brotitis: es realizada cuando las condiciones del clima lo requieran, es decir, cuando existan precipitaciones, dado que cuando precipita es muy probable que la planta se infecte con hongos, por lo que se debe aplicar algún fungicida.

- Control de arañitas: las arañitas son una plaga que se reproduce rápidamente. El control de arañitas consiste en la aplicación de pesticidas sobre la planta. 
- Cosecha de frambuesas Meeker y Heritage: existen varios tipos de frambuesas en Chile, entre las más producidas en la región se encuentran la tipo Meeker (una sola flor) y la de tipo Heritage (dos flores).

Ahora bien, en cuanto a los costos involucrados en este proceso productivo, dado a que en esta provincia existe una clara dispersión por parte de los productores de frambuesas, razón por la cual es difícil determinar una homogeneidad en la determinación de sus costos o del manejo que mantienen, sobre todo los pequeños productores de la zona. Frente a este panorama, es necesario conocer entonces la información, las técnicas y métodos en que ellos basan su sistema de costeo. De esta forma contribuiremos a resolver en parte el complejo y variable entorno económico, que presenta este mercado en la provincia de Nuble. En este contexto, se entiende por Pequeño Productor Agrícola a aquel que explota una superficie no superior a 12 hectáreas de riego básico, cuyos activos no superen el equivalente a 3.500 unidades de fomento, que su ingreso provenga principalmente de la explotación agrícola y que trabaje directamente la tierra, cualquiera sea su régimen de tenencia. Según la Fundación para la Innovación Agraria (FIA, 2004) alrededor de un $70 \%$ de la producción de frambuesas se encuentra mayoritariamente en manos de pequeños productores agrícolas. En el país, el promedio de superficie cultivada por productor es de 1 a 2 hectáreas. Asimismo, se estima que esta es una actividad que demanda una gran cantidad de mano de obra, especialmente en época de cosecha, empleando alrededor de 75.000 personas.

En este escenario, la gestión de costos de los pequeños productores de la zona, resulta de vital importancia debido a que si esta es realizada de manera adecuada permitirá mejorar los índices de rentabilidad de los mismos y por ende potenciar la economía regional.

En base a lo anteriormente mencionado y dado la poca información que se existe acerca del tema, surge entonces la siguiente pregunta de investigación ¿Los pequeños productores de frambuesas de la provincia de Ñuble, están determinando adecuadamente sus costos de producción?, por lo cual, el presente trabajo tuvo como objetivo analizar el sistema de registro de costos utilizado por los pequeños productores de frambuesas, usuarios del Instituto de Desarrollo Agropecuario (INDAP) de la provincia de Ñuble con el fin de proponer un sistema de administración de costos de fácil comprensión y utilización para éstos. Este trabajo considera en un primer apartado los aspectos metodológicos del estudio y posteriormente los resultados del mismo, donde en primer lugar se muestra el diagnóstico realizado y luego la propuesta de sistema de costeo.

\section{METODOLOGÍA, MATERIAL Y MÉTODOS}

Desde el punto de vista metodológico se utilizaron técnicas pertenecientes al paradigma de investigación cuantitativo y cualitativo, el instrumento a utilizar fue una encuesta debidamente validada a través del juicio de expertos, también se utilizaron técnicas como entrevistas en caso de tener dudas con algunos resultados, observación y levantamiento de información en terreno. El instrumento cuenta con 
preguntas cerradas por cada etapa del proceso productivo de la frambuesa (12 etapas). El universo a considerar son 50 pequeños productores de frambuesa que son usuarios del Instituto de Desarrollo Agropecuario (INDAP) y que tienen alianza productiva con la empresa ALIFRUT. El tamaño de la muestra considera a 33 de estos pequeños productores considerando un nivel de significancia del $90 \%$ y un margen de error del $10 \%$. Se realizó un muestreo aleatorio por tipo de manejo productivo y por zona geográfica y se supone una distribución normal de la población. Para el estudio se analizaron los resultados de 16 productores de tipo convencional y 17 de tipo orgánico, todos ubicados geográficamente en la provincia de Ñuble, Chile.

\section{RESULTADOS}

\section{Diagnóstico al proceso de producción de frambuesas:}

A continuación se muestran los resultados de las encuestas aplicadas a 33 pequeños productores de la Provincia de Ñuble, de los cuales 16 tienen producción de tipo convencional y 17 de tipo orgánica. La primera pregunta busca determinar la realización o no de cada una de las etapas por los productores sujetos de estudio (Ver Tabla 1).

\section{Tabla 1: Realización de la etapa}

\begin{tabular}{|l|r|r|r|r|r|r|r|r|}
\hline \multicolumn{1}{|c|}{ ETAPA } & \multicolumn{3}{c|}{ Producción convencional } & \multicolumn{3}{c|}{ Producción orgánica } \\
& \multicolumn{2}{|c|}{ SI } & \multicolumn{2}{c|}{ NO } & \multicolumn{2}{c|}{ SI } & \multicolumn{2}{c|}{ NO } \\
\hline Poda & 16 & $100 \%$ & 0 & $0 \%$ & 17 & $100 \%$ & 0 & $0 \%$ \\
Control de larva & 11 & $69 \%$ & 5 & $31 \%$ & 13 & $76 \%$ & 4 & $24 \%$ \\
Fertilización & 16 & $100 \%$ & 0 & $0 \%$ & 17 & $100 \%$ & 0 & $0 \%$ \\
Fertilización con nitrógeno & 12 & $75 \%$ & 4 & $25 \%$ & 12 & $71 \%$ & 5 & $29 \%$ \\
Control de maleza & 16 & $100 \%$ & 0 & $0 \%$ & 17 & $100 \%$ & 0 & $0 \%$ \\
Control de burritos & 6 & $38 \%$ & 10 & $62 \%$ & 1 & $6 \%$ & 16 & $94 \%$ \\
Fertilización foliar & 13 & $81 \%$ & 3 & $19 \%$ & 17 & $100 \%$ & 0 & $0 \%$ \\
Control de enfermedades & 7 & $43 \%$ & 9 & $57 \%$ & 11 & $65 \%$ & 6 & $35 \%$ \\
Control de brotritis & 6 & $38 \%$ & 10 & $62 \%$ & 7 & $41 \%$ & 10 & $59 \%$ \\
Control de arañitas & 7 & $43 \%$ & 9 & $57 \%$ & 3 & $18 \%$ & 14 & $82 \%$ \\
Cosecha Meeker & 13 & $81 \%$ & 3 & $19 \%$ & 13 & $76 \%$ & 4 & $24 \%$ \\
Cosecha Heritage & 15 & $94 \%$ & 1 & $6 \%$ & 17 & $100 \%$ & 0 & $0 \%$ \\
\hline
\end{tabular}

Como se observa en la Tabla 1, en promedio un $72 \%$ de los productores convencionales realizan la mayoría de las etapas y un $71 \%$ en el caso de los productores orgánicos. Siendo las etapas más realizadas por ambos productores las etapas de Poda, Fertilización, Control de Maleza, y Fertilización foliar. La especie más cosechada es la de tipo Heritage y las etapas menos realizadas son control de burritos, control de brotitis, control de enfermedades y control de arañitas, en ambos casos. Estas últimas etapas tienen en común que solo deben realizarse en caso de que se encuentren plagas, deterioro por lluvias 0 enfermedades en la planta de frambuesa, no siendo necesario realizarla en todos los casos. Sin 
embargo, el control de enfermedades en el caso de los productores orgánicos se realiza por una mayor cantidad de productores.

La segunda pregunta realizada al productor fue si llevaba algún tipo de registro sobre la etapa, ya sea sobre el costo incurrido, la mano de obra o el tiempo que utilizó para realizarla. En relación a lo anterior, como se observa en la Tabla 2 hay un alto porcentaje de productores que llevan registros en las distintas etapas, un $77 \%$ en el caso de los productores convencionales y un $93 \%$ en el caso de los productores orgánicos, (Ver Tabla 2).

\section{Tabla 2: Registro en cada etapa}

\begin{tabular}{|l|r|r|r|r|r|r|r|r|}
\hline \multirow{2}{*}{\multicolumn{1}{|c|}{ ETAPA }} & \multicolumn{3}{c|}{$\begin{array}{c}\text { Producción } \\
\text { Convencional }\end{array}$} & \multicolumn{3}{c|}{ Producción orgánica } \\
\cline { 2 - 9 } & \multicolumn{2}{|c|}{ SI } & \multicolumn{2}{c|}{ N0 } & \multicolumn{2}{c|}{ SI } & \multicolumn{2}{|c|}{ N0 } \\
\hline Poda & 12 & $75 \%$ & 4 & $25 \%$ & 16 & $94 \%$ & 1 & $6 \%$ \\
Control de larva & 6 & $55 \%$ & 5 & $45 \%$ & 11 & $85 \%$ & 2 & $15 \%$ \\
Fertilización & 8 & $50 \%$ & 8 & $50 \%$ & 17 & $100 \%$ & 0 & $0 \%$ \\
Fertilización con nitrógeno & 9 & $75 \%$ & 3 & $25 \%$ & 12 & $100 \%$ & 0 & $0 \%$ \\
Control de maleza & 12 & $75 \%$ & 4 & $25 \%$ & 14 & $82 \%$ & 3 & $18 \%$ \\
Control de burritos & 5 & $83 \%$ & 1 & $17 \%$ & 1 & $100 \%$ & 0 & $0 \%$ \\
Fertilización foliar & 10 & $77 \%$ & 3 & $23 \%$ & 17 & $100 \%$ & 0 & $0 \%$ \\
Control de enfermedades & 5 & $71 \%$ & 2 & $29 \%$ & 10 & $91 \%$ & 1 & $9 \%$ \\
Control de brotritis & 6 & $100 \%$ & 0 & $0 \%$ & 7 & $100 \%$ & 0 & $0 \%$ \\
Control de arañitas & 5 & $71 \%$ & 2 & $29 \%$ & 2 & $67 \%$ & 1 & $33 \%$ \\
Cosecha Meeker & 13 & $100 \%$ & 0 & $0 \%$ & 13 & $100 \%$ & 0 & $0 \%$ \\
Cosecha Heritage & 14 & $93 \%$ & 1 & $7 \%$ & 17 & $100 \%$ & 0 & $0 \%$ \\
\hline
\end{tabular}

Respecto a qué registra cada agricultor en cada una de las etapas, se pudo observar que este es bastante vago, siendo los registros más frecuentes el pago de sueldos a los trabajadores, la duración de las etapas y la dosis, tipo de producto aplicado y el precio de este en el caso de los productores convencionales, (Ver Tabla 3).

Tabla 3: Tipo de registro llevado en cada etapa productores convencionales

\begin{tabular}{|l|c|c|c|c|c|c|}
\hline Etapa & $\begin{array}{l}\text { Sueldos } \\
\text { pagados }\end{array}$ & Duración & $\begin{array}{l}\text { Dosis, } \\
\text { producto } \\
\text { y precio }\end{array}$ & $\begin{array}{l}\text { Cantidad } \\
\text { cosechada }\end{array}$ & $\begin{array}{l}\text { Cantidad de } \\
\text { trabajadores }\end{array}$ & Ninguno \\
\hline Poda & 4 & 5 & 5 & & 3 & 4 \\
Control de larva & 1 & 2 & 6 & & & 5 \\
Fertilización & & 1 & 7 & & 1 & 3 \\
Fertilización con & & 1 & & & & 4 \\
nitrógeno & 5 & 7 & 3 & & & 1 \\
Control de maleza & 1 & 1 & 7 & & & 3 \\
Control de burrito & 3 & & 2 & & & 2 \\
Fertilización foliar & 2 & 1 & 2 & & & \\
Control de & 2 & 1 & 3 & & & 2 \\
enfermedades & 2 & & 3 & 5 & & 1 \\
Control de brotritis & 2 & & 6 & & \\
Control de arañitas & 2 & & & & \\
Cosecha Meeker & 8 & & & & \\
Cosecha Heritage & 8 & & &
\end{tabular}


En el caso de los productores orgánicos existe un mayor nivel de registro dado que este es exigido por organismos certificadores, siendo registrado además de los sueldos pagados a trabajadores, la duración, dosis, producto aplicado y precio de éste, los desembolsos realizados en cada etapa, (Ver Tabla 4).

Tabla 4: Tipo de registro llevado en cada etapa productores orgánicos

\begin{tabular}{|c|c|c|c|c|c|c|}
\hline Etapa & $\begin{array}{l}\text { Sueldos } \\
\text { pagados }\end{array}$ & Duración & $\begin{array}{l}\text { Dosis, } \\
\text { producto } \\
\text { y precio }\end{array}$ & $\begin{array}{l}\text { Cantidad } \\
\text { cosechada }\end{array}$ & $\begin{array}{l}\text { Gastos } \\
\text { incurridos }\end{array}$ & Ninguno \\
\hline Poda & 5 & 9 & & & 2 & 1 \\
\hline Control de larva & & 1 & 9 & & 1 & 2 \\
\hline Fertilización & 1 & 2 & 12 & & 2 & \\
\hline $\begin{array}{l}\text { Fertilización con } \\
\text { nitrógeno }\end{array}$ & & 1 & 10 & & 1 & \\
\hline Control maleza & 3 & 9 & & & 2 & 3 \\
\hline Control burrito & & 1 & & & & \\
\hline Fertilización foliar & & 5 & 11 & & 1 & \\
\hline $\begin{array}{l}\text { Control de } \\
\text { enfermedades }\end{array}$ & 3 & 2 & 5 & & & 1 \\
\hline Control brotritis & & 3 & 4 & & & \\
\hline Control arañitas & & 1 & 1 & & & 1 \\
\hline Cosecha Meeker & 7 & & & 6 & & \\
\hline Cosecha Heritage & 9 & & & 8 & & \\
\hline
\end{tabular}

El tiempo que se requiere para el desarrollo de cada etapa es distinto y está relacionado con la cantidad de hectáreas que posee cada agricultor. Los resultados arrojados por esta pregunta, refleja que las etapas que requieren menos tiempo para ser realizadas son, fertilización con nitrógeno, fertilización foliar, control de enfermedades y control de brotitis en ambos tipos de productores, las que requieren menos de una semana para ser desarrolladas. Entre estas etapas es común que el tiempo requerido para su desarrollo no pase más allá de unos tres días aproximadamente, mientras que las etapas que conllevan más tiempo son la cosecha, las que se realizan durante el verano, con una duración aproximada de unos 5 meses para la variedad de Heritage y unos 45 días en la variedad Meeker, (Ver Tabla 5).

Tabla 5: Duración de cada etapa en la producción de frambuesa

\begin{tabular}{|c|c|c|c|c|c|c|c|c|c|c|c|c|}
\hline \multirow[b]{2}{*}{ Etapa } & \multirow[b]{2}{*}{ Productor } & \multicolumn{10}{|c|}{$N^{\circ}$ de Semanas } & \multirow{2}{*}{ 票 } \\
\hline & & $0-1$ & $2-3$ & 4-5 & 6-7 & $8-9$ & $12-13$ & $16-17$ & 18-19 & $20-21$ & $22-23$ & \\
\hline \multirow{2}{*}{ Poda } & Convencional & & 6 & 7 & & 3 & & & & & & 16 \\
\hline & Orgánico & & 8 & 6 & & 3 & & & & & & 17 \\
\hline \multirow{2}{*}{ Control de larva } & Convencional & 9 & 2 & & & & & & & & & 11 \\
\hline & Orgánico & 12 & & 1 & & & & & & & & 13 \\
\hline \multirow{2}{*}{ Fertilización } & Convencional & 12 & 4 & & & & & & & & & 16 \\
\hline & \begin{tabular}{|l|} 
Orgánico \\
\end{tabular} & 14 & 3 & & & & & & & & & 17 \\
\hline \multirow{2}{*}{$\begin{array}{l}\text { Fertilización con } \\
\text { nitrógeno }\end{array}$} & Convencional & 12 & & & & & & & & & & 12 \\
\hline & Orgánico & 12 & & & & & & & & & & 12 \\
\hline
\end{tabular}




\begin{tabular}{|c|c|c|c|c|c|c|c|c|c|c|c|c|}
\hline \multirow{2}{*}{ Etapa } & \multirow{2}{*}{ Productor } & \multicolumn{10}{|c|}{$\mathrm{N}^{\circ}$ de Semanas } & \multirow{2}{*}{ 퓽 } \\
\hline & & $0-1$ & $2-3$ & $4-5$ & $6-7$ & $8-9$ & $12-13$ & $16-17$ & 18-19 & $20-21$ & $22-23$ & \\
\hline \multirow{2}{*}{$\begin{array}{l}\text { Control de } \\
\text { maleza }\end{array}$} & Convencional & 8 & 8 & & & & & & & & & 16 \\
\hline & Orgánico & 2 & 4 & 3 & 1 & 2 & 4 & 1 & & & & 17 \\
\hline \multirow{2}{*}{$\begin{array}{l}\text { Control de } \\
\text { burrito }\end{array}$} & Convencional & 5 & 1 & & & & & & & & & 6 \\
\hline & Orgánico & 1 & & & & & & & & & & 1 \\
\hline \multirow{2}{*}{$\begin{array}{l}\text { Fertilización } \\
\text { foliar }\end{array}$} & Convencional & 13 & & & & & & & & & & 13 \\
\hline & Orgánico & 15 & 2 & & & & & & & & & 17 \\
\hline \multirow{2}{*}{$\begin{array}{l}\text { Control de } \\
\text { enfermedades }\end{array}$} & Convencional & 7 & & & & & & & & & & 7 \\
\hline & Orgánico & 11 & & & & & & & & & & 11 \\
\hline \multirow{2}{*}{$\begin{array}{l}\text { Control de } \\
\text { brotritis }\end{array}$} & Convencional & 6 & & & & & & & & & & 6 \\
\hline & Orgánico & 7 & & & & & & & & & & 7 \\
\hline \multirow{2}{*}{$\begin{array}{l}\text { Control de } \\
\text { arañitas }\end{array}$} & Convencional & 6 & 1 & & & & & & & & & 7 \\
\hline & Orgánico & 3 & & & & & & & & & & 3 \\
\hline \multirow{2}{*}{$\begin{array}{l}\text { Cosecha } \\
\text { Meeker }\end{array}$} & Convencional & & & 4 & 4 & 5 & & & & & & 13 \\
\hline & Orgánico & & & 10 & & 3 & & & & & & 13 \\
\hline \multirow{2}{*}{$\begin{array}{l}\text { Cosecha } \\
\text { Heritage }\end{array}$} & Convencional & & & & & & 1 & & 4 & & 10 & 15 \\
\hline & Orgánico & & & & & & 3 & 3 & & 10 & 1 & 17 \\
\hline
\end{tabular}

Respecto a la mano de obra se observa que en las etapas de poda y cosecha es donde mayoritariamente se utiliza mano de obra temporal, para ambos productores, (Ver Tabla 6).

Tabla 6: Mano de obra utilizada en cada etapa

\begin{tabular}{|c|c|c|c|c|c|}
\hline Etapa & \begin{tabular}{|l|} 
Productor \\
\end{tabular} & Dueño & Contratado & Temporeros & Total \\
\hline \multirow{2}{*}{ Poda } & Convencional & 4 & 1 & 11 & 16 \\
\hline & Orgánico & 7 & 2 & 8 & 17 \\
\hline \multirow{2}{*}{ Control de larva } & Convencional & 7 & 2 & 2 & 11 \\
\hline & Orgánico & 6 & 2 & 5 & 13 \\
\hline \multirow{2}{*}{ Fertilización } & Convencional & 7 & 3 & 6 & 16 \\
\hline & Orgánico & 8 & 3 & 6 & 17 \\
\hline \multirow{2}{*}{ Fertilización con nitrógeno } & Convencional & 7 & 2 & 3 & 12 \\
\hline & Orgánico & 6 & 2 & 4 & 12 \\
\hline \multirow{2}{*}{ Control de maleza } & Convencional & 4 & 3 & 9 & 16 \\
\hline & Orgánico & 7 & 3 & 7 & 17 \\
\hline \multirow{2}{*}{ Control de burrito } & Convencional & 1 & 1 & 4 & 6 \\
\hline & Orgánico & 1 & & & 1 \\
\hline \multirow{2}{*}{ Fertilización foliar } & Convencional & 5 & 2 & 6 & 13 \\
\hline & Orgánico & 8 & 3 & 6 & 17 \\
\hline \multirow{2}{*}{ Control de enfermedades } & Convencional & 4 & 1 & 2 & 7 \\
\hline & Orgánico & 8 & & 3 & 11 \\
\hline \multirow{2}{*}{ Control de brotritis } & Convencional & 3 & & 3 & 6 \\
\hline & Orgánico & 4 & & 3 & 7 \\
\hline \multirow{2}{*}{ Control de arañitas } & Convencional & 3 & 2 & 2 & 7 \\
\hline & Orgánico & 3 & & & 3 \\
\hline \multirow{2}{*}{ Cosecha Meeker } & Convencional & & & 13 & 13 \\
\hline & Orgánico & & & 13 & 13 \\
\hline \multirow{2}{*}{ Cosecha Heritage } & Convencional & & & 15 & 15 \\
\hline & \begin{tabular}{|l|} 
Orgánico \\
\end{tabular} & & & 17 & 17 \\
\hline
\end{tabular}

En lo que respecta al uso de maquinarias, de acuerdo a los resultados se observa 
que gran parte de las actividades no requieren de maquinarias sino más bien, son realizadas manualmente por personas, y en los casos en estas son requeridas son mayoritariamente de propiedad del dueño en ambos tipo de producción, tanto convencional como orgánica, es importante señalar que en la etapa de cosecha de ambas variedades de frambuesas tanto tipo Meeker como Heritage, en ambos tipo de productores, convencionales y orgánicos, son utilizadas mayoritariamente maquinarias arrendadas, (Ver Tabla 7).

Tabla 7: Maquinaria utilizada en cada etapa

\begin{tabular}{|c|c|c|c|c|c|c|}
\hline Etapa & Productor & Arrendada & Propia & $\begin{array}{l}\text { Propia y } \\
\text { arrendada }\end{array}$ & \begin{tabular}{|l|} 
No \\
utiliza
\end{tabular} & Total \\
\hline \multirow{2}{*}{ Poda } & Convencional & 2 & 14 & & & 16 \\
\hline & Orgánico & & 17 & & & 17 \\
\hline \multirow{2}{*}{ Control de larva } & Convencional & & & & 11 & 11 \\
\hline & Orgánico & & 13 & & & 13 \\
\hline \multirow{2}{*}{ Fertilización } & Convencional & & & & 16 & 16 \\
\hline & Orgánico & & & & 17 & 17 \\
\hline \multirow{2}{*}{$\begin{array}{l}\text { Fertilización con } \\
\text { nitrógeno }\end{array}$} & Convencional & & & & 12 & 12 \\
\hline & Orgánico & 4 & 8 & & & 12 \\
\hline \multirow{2}{*}{ Control de maleza } & Convencional & & 16 & & & 16 \\
\hline & Orgánico & & 14 & 3 & & 17 \\
\hline \multirow{2}{*}{ Control de burrito } & Convencional & & & & 6 & 6 \\
\hline & Orgánico & & & & 1 & 1 \\
\hline \multirow{2}{*}{ Fertilización foliar } & Convencional & 1 & 12 & & & 13 \\
\hline & Orgánico & & 17 & & & 17 \\
\hline \multirow{2}{*}{$\begin{array}{l}\text { Control de } \\
\text { enfermedades }\end{array}$} & Convencional & & 3 & & 4 & 7 \\
\hline & Orgánico & & 11 & & & 11 \\
\hline \multirow{2}{*}{ Control de brotritis } & Convencional & & & & 6 & 6 \\
\hline & Orgánico & & 7 & & & 7 \\
\hline \multirow{2}{*}{ Control de arañitas } & Convencional & & & & 7 & 7 \\
\hline & Orgánico & & & & 3 & 3 \\
\hline \multirow{2}{*}{ Cosecha Meeker } & Convencional & 7 & 4 & 1 & 1 & 13 \\
\hline & Orgánico & 6 & 1 & 6 & & 13 \\
\hline \multirow{2}{*}{ Cosecha Heritage } & Convencional & 8 & 5 & 1 & 1 & 15 \\
\hline & Orgánico & 8 & 2 & 7 & & 17 \\
\hline
\end{tabular}

\section{Determinación de puntos críticos:}

En resumen, se desprende que en la producción de frambuesas (tipo convencional), los agricultores tienden más a no llevar registros sobre las etapas de la producción, como son la cantidad utilizada y la cantidad de mano de obra ocupada. Cabe destacar que en este tipo de agricultores, los costos de producción son de poca relevancia para ellos, llevando una estimación mental sobre el costo incurrido para desarrollar cada etapa de la producción.

Lo más registrado por los agricultores convencionales, es la cantidad ocupada de algún fertilizante 0 producto que se debe aplicar a la planta, y por supuesto el nombre del producto.

En las etapas en que los agricultores llevan mayor control sobre los costos, es en las etapas finales del proceso, en donde se cosecha la fruta, la mayoría lleva registro sobre la cantidad de cajas cosechadas que cada trabajador toma, para 
luego desembolsar el valor de la mano de obra que conlleva la cosecha de la frambuesa. Otro punto importante a destacar es la mano de obra, el recurso más importante para la producción de esta fruta, la que mayoritariamente en la producción es de carácter temporal, por lo que muchos de los agricultores no establecen algún contrato de trabajo para sus empleados, conllevando también a que muchas de las labores dentro de la producción la realicen los propios dueños. Respecto a los productores orgánicos, el nivel de realización de las etapas es más alto en las etapas principales, las cuales son control de maleza, fertilización foliar, poda y cosecha, ya que estas deben realizarse en todas las temporadas. La etapa de cosecha es la que acumula la mayor parte del costo, por ende es muy importante el registro de dicha etapa. Como se pudo observar en la mayoría de las etapas los agricultores llevan un registro de las actividades que realizan, no obstante el nivel y/o tipo de registro que llevan es muy básico a nivel administrativo contable, registrando principalmente, el pago de los trabajadores, el productos aplicados, duración de las etapas, cantidades cosechadas, pero en muy pocos casos, los desembolsos asociados.

Cabe destacar que los productores orgánicos llevan registro de las actividades que realizan tanto por criterio propio y por petición de la certificadora, como Bio Certificadora Servicio Limitada (BCS) quien solicitan meramente registros en el área productiva ya sea en dosis y producto utilizado y la fecha en que se aplica, pero la mayoría de los productores no sabe cuánto gasta en cada etapa. Los registros exigidos por la certificadora son solo para corroborar que los productores estén utilizando los productos permitidos para que su predio sea autorizado como orgánico, sin embargo, en el caso de los productores convencionales ellos no tienen obligación de mantener ningún registro, por ende la gran mayoría lleva un registro muy vago o simplemente no lleva ningún tipo de registro.

Por lo general, los registros que llevan los productores orgánicos y los convencionales son muy similares, ambos registran la dosis del producto utilizado en cada etapa, ambos llevan un nulo o muy básico nivel de registro de los desembolsos que realizan durante las etapas de pre-cosecha, dándole a la etapa de cosecha el mayor nivel de registro a nivel monetario ya que en este ellos ven el mayor gasto producido el pago a los trabajadores y también se registra los ingresos que ellos perciben. En sí, ambos procesos son iguales solo se diferencian en los productos que se les permiten aplicar y en el valor que implica cada uno, un claro ejemplo está en el control de maleza, los productores convencionales están en total facultad de aplicar cualquier tipo de herbicida para así poder controlar el crecimiento de las malezas que se encuentren en su predio, esto implica un costo menor ya que el herbicida es aplicado por una sola persona y esta aplicación toma poco tiempo, en el caso de los productores orgánicos ellos no están autorizados a utilizar herbicidas los que hace que el trabajo se vuelve constante durante toda la etapa de post-cosecha lo que la hace de larga duración.

Finalmente, es posible deducir que los productores solo registran lo que se les está obligado a registrar y lo que no necesariamente dice relación con el costo sino más bien con el área productiva, sin embargo la gran mayoría de los productores tienen cursos relacionados con la administración y contabilidad, lo que genera una gran 
ventaja en el planteamiento de un sistema de costos para que ellos puedan generar una visión clara de sus movimientos de dinero y obtener un resultado claro en cada etapa y al final de su producción.

\section{Propuesta de sistema de costeo}

Como ya se mencionó anteriormente, la producción de frambuesas se lleva a cabo fundamentalmente en procesos, es decir por etapas. Es por eso, que el agricultor debe saber y analizar todo lo que conlleva monetariamente cada etapa para así poder llevar un mayor control sobre los costos en que él incurre durante el proceso. El sistema de costeo elegido debe clasificar, registrar y agrupar los egresos de tal forma que le permita al interesado conocer el costo unitario de cada proceso y producto. Esto solo se puede realizar mediante el diseño de un sistema de costeo adecuado para la actividad, por lo tanto, por la naturaleza de la actividad de la producción de frambuesas, SISTEMA DE COSTEO POR PROCESOS es el más adecuado para tener una clara visión de los costos generados y por consiguiente su valor unitario, ya que el producto en este caso son idénticos. La principal razón es que la producción de frambuesas es que esta se encuentra dividida claramente por etapas y se generan productos homogéneos al final de la producción.

Asimismo se propone un registro de COSTOS HISTÓRICOS, dado que los productores podrán determinar de manera más fácil, ya que solo se necesita que sean costos obtenidos dentro del periodo de fabricación del producto o etapa correspondiente, este procedimiento consiste en la anotación de los gastos incurridos en los materiales utilizados, la mano de obra empleada y los gastos indirectos de fabricación que se acumulan durante la etapa o producción.

Para que el agricultor pueda llegar a determinar los costos de buena manera, se entregan las siguientes recomendaciones:

- Llevar un orden de todas las facturas y boletas de los gastos que el agricultor incurra en las diferentes etapas de la producción, e ir asociando cada una de ellas a las distintas etapas, para así poder registrar posteriormente los valores a la planilla de costos.

- Llevar una planilla detallada, en la cual el agricultor pueda ir registrando cada desembolso que le conlleve la elaboración de esa etapa, en donde éste registrará el material ocupado, las horas de mano de obra requeridas y todos los otros gastos que el agricultor incurra para desarrollar la etapa.

- Determinar el precio que el agricultor desembolsará por la hora de mano de obra requerida para las distintas etapas de la producción del producto, y llevar el registro de las horas que se requerirán en cada etapa en la mencionada planilla.

Respecto a los costos indirectos serán asignados con una tasa relativa a la duración de cada etapa, la cual hará que sea más fácil su cálculo para los pequeños

agricultores. La duración de cada etapa será relativa a la extensión del campo, la cantidad de trabajadores que utiliza en cada una, los montos que se asignen a cada 
etapa serán en su mayoría asignados a la etapa de cosecha ya que en ella es la que se encuentra mayor tiempo invertido es en esta, asimismo, este es un registro ya realizado por los agricultores, por lo cual, no les significará una carga de trabajo administrativo adicional.

\section{CONCLUSIONES}

A raíz de este estudio es posible concluir que existen diferencias muy claras en el tratamiento que aplican a la información obtenida entre los productores orgánicos y los productores tradicionales de frambuesa, dado que los primeros en su gran mayoría mantienen un registro de las actividades realizadas detallando su fecha y los productos utilizados, sin embargo, esta información no es utilizada para el posterior cálculo de los costos finales de la producción, limitándose a generar solo información de proceso, la cual aún sigue siendo vaga.

Del mismo modo, se concluye que los pequeños productores de frambuesas de la Provincia de Ñuble no mantienen un sistema estandarizado para el manejo de sus costos, lo que es una falencia grave, más aún si se considera el constante crecimiento que se mantiene de la superficie plantada y el nivel de exportación que presenta este mercado.

El no contar con un sistema de costos, que les proporcione información detalle del costo de producción, y la rentabilidad de sus productos, están en riesgo de perdidas, dado que sus precios de venta son inestables y depende generalmente del mercado externo, siendo entonces, la única alternativa para rentabilizar sus productos, la optimización de sus costos internamente, lo cual es muy difícil cuando no se tiene claridad de los costos en que incurre en el proceso de producción en general, 0 específicamente en cada una de las etapas de este proceso, que les permita tomar decisiones, gestionar o crecer como empresa.

Asimismo, estos productores demostraron un conocimiento adquirido mediante los contantes cursos implementados por INDAP y la empresa frutícola Alifrut en materias administrativas y de gestión contable, sin embargo, no hacen uso de estos conocimientos para llevar un control de su propia empresa.

Finalmente, es posible recomendar a los pequeños productores un sistema de costos que les permitirá con información detallada de cada uno de los costos incurridos en el proceso de fabricación, de fácil uso, comprensible y adecuado a las características propias de los productores, lo cual les permitirá tomar decisiones informados y mejorar sus rendimientos económicos y financieros. 


\section{BIBLIOGRAFÍA}

- Biblioteca del congreso nacional de Chile. (2012). Ley 18.910. Recuperado de http://www.leychile.cl/Navegar?idNorma=30282.

- Rebolledo, José Manuel. (24-05-2012). Desde Chillán potencian berries en todo el país. Diario La Discusión. Recuperado de http://h.ladiscusion. cl/index.php/archivos/12395-desde-chillan-potencian-berries-en-todo-elpais.

- Fundación para la Innovación Agrícola. (2012). Agenda de los berries. Recuperado de http://www.fia.cl/download/estudios-fia/innovacionagraria-territorial/Agenda\%20Biobi\%CC\%810.pdf

- Gutiérrez, L. (2011). Productores de arándanos y frambuesas son capacitados en control de plagas y mitigación de enfermedades en Chillán. Recuperado de http://www.copevaldesarrolla.cl/web/index.php/ noticias/266-productores-de-hortalizas-participan-de-dia-de-campo-enalternativas-al-control-de-plagas-y-enfermedades-horticolas

- Horngren, Sundem y Stratton. (2006). Contabilidad administrativa, $13^{\circ}$ edición, México: Pearson Educación.

- INDAP. (2012). Resultados de evaluación de variedades y manejos productivos de la frambuesa en Chile. Recuperado de http://www. indap.gob.cl/Docs/Documentos/Fruticultura/Frambuesa/resultados_de_ evaluacion_de_variedades_y_manejos_productivos_de_la_frambuesa_ en_chile.PDF.

- INDAP. (2011). Estrategias Regionales de Competitividad por Rubro "BERRIES REGIÓN DEL MAULE". Recuperado de http://beta1.indap.cl/ Docs/Documentos/Estrategias\%20Regionales\%20Competitividad\%20 por\%20Rubro/Estrategias\%20Regionales $\% 20 \mathrm{y} \% 20$ Planes $\% 20$ Nacionales\%202007/Regi\%C3\%B3n\%20del\%20Maule/Berries-VIIR_ EstrategiasRegionalesXRubro.pdf.

- INE. (2012). Informe de empleo. Recuperado de http://www.inebiobio. cl/archivos/files/Informe\%20Emple0\%20\%C3\%91uble\%20EneMar\%20 2012.pdf.

- Ortega, R. (2012). Fertilización Foliar. Otra exitosa forma de nutrir a las plantas. Recuperado de http://www.fertilizando.com/articulos/ Fertilizacion\%20Foliar\%20-\%200tra\%20forma\%20exitosa.asp.

- Prochile. (2011). Informativo de Mercado de Berries Frescos y Congelados 
2011/12. Recuperado de http://www.prochile.cl/regiones_pro/archivos/ region_VII/boletin_2012/boletin_06.pdf.

- Quezada, F. (2006). Análisis del sector hortofrutícola provincia de Ñuble. Recuperado de http://www.tallerdeempleo.cl/informes/ter026b2.pdf.

- Sánchez, E. (2006). Diagnóstico y proyección de la producción de arándanos en la zona sur de Chile. Recuperado de http://cybertesis.uach. cl/tesis/uach/2006/fas211d/doc/fas211d.pdf.

- Seragro. (2007). Todo sobre los berries de Chile. Recuperado de http:// seragro. $\mathrm{cl} / ? \mathrm{a}=1343$.

- Serplac, (2008). Pobreza, empleo y educación, recuperado en octubre del 2012, de http://www.gorebiobio.cl/Documentos/Genero/Cuadernillo\%20 2\%20final_c.pdf

- Vera, M. (2012). El dañino burrito que ataca a los berries. Recuperado de http://camposureno.wordpress.com/2010/05/17/el-danino-burrito-queataca-a-los-berries/.

- Zamora, D. (2012). Control de araña roja en frambuesas. Recuperado de http://nutricarezamora.blogspot.com/2012/08/control-de-arana-roja-enframbuesa.htm 